\title{
Идентификация пор ультрафильтрационных мембран на основе электронно-микроскопических исследований
}

\author{
(С) 2021 Лазарев С.И. ${ }^{1}$, Коновалов Д.Н. ${ }^{1}$, Ковалев С.В. ${ }^{1,2}$, Рыжкин В.Ю. ${ }^{1}$, \\ Полянский К.К. ${ }^{3}$, Ковалева О.А. ${ }^{1,2}$ \\ ${ }^{1}$ Тамбовский государственный технический университет, Тамбов \\ ${ }^{2}$ Тамбовский государственный университет имени Г.Р. Державина, Тамбов \\ ${ }^{3}$ Воронежский филиал Российского экономического университета имени Г.В. Плеханова, Воронеж
}

Поступила в редакцию 18.02.2021 г.

DOI: $10.17308 /$ sorpchrom.2021.21/3470

В работе проанализированы методы, способы, приемы и прикладные программы для идентификации пор в полимерных мембранах. На основе сравнения достоинств и недостатков методов предложен подход к разработке программной реализации исследования пор полимерных полупроницаемых мембран. Объектами исследования служили ультрафильтрационные мембраны вида УАМ-50, УАМ100, УПМ-К, УПМ-100, выбор которых обеспечен высокой задерживающей способностью, хорошей производительностью и наибольшей применяемостью в промышленной практике. Приведена методика по расчету коэффициента засоренности мембран, которая позволяет определить срок эффективной работы ультрафильтрационных мембран, элементов и установок при баромембранном и электробаромембранном разделении, концентрировании и очистке промышленных растворов и стоков. Выделенные участки ультрафильтрационных мембран УАМ-50, УАМ-100, УПМ-К, УПМ-100 площадью 1000000

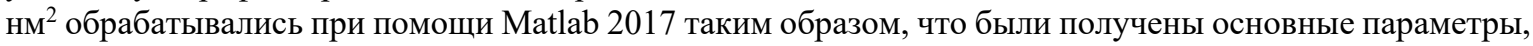
такие как средний диаметр засоренности (диаметры пор и коэффициент засоренности мембран). При обработке больших массивов данных по средним диаметрам пор и коэффициенту засоренности мембран использовался ПК, который позволил снизить и рассчитать погрешность выполненных измерений при помощи стандартных методов математической статистики. Расчет коэффициента засоренности мембран производили при помощи программы, изучающей описание основных функций imaging processing toolbox. Разработанный метод существенно сокращает время эксперимента и позволяет автоматизировано рассчитывать количество объектов, среднюю площадь, диаметр пор на сорбционной поверхности. Метод, сочетающий электронно-микроскопические исследования, обработку изображений Otsu's method, программную реализацию в Matlab 2017, дают возможность получить достоверные и воспроизводимые данные по морфологии поверхности ультрафильтрационных мембран УАМ-50, УАМ-100, УПМ-К, УПМ-100, опирающихся на статистическую обработку большой выборки данных, полученных в результате электронно-микроскопических исследований. Анализ экспериментальных данных, полученных автоматизированным методом, показал, что средняя площадь объекта наименьшая для мембраны УПМ-К и наибольшая для мембраны УАМ-50, а средний диаметр пор поверхности исследуемых мембран находится в интервале от 51 до 60 нм, что сопоставимо с литературными данными, полученных другими методами. При этом коэффициент засоренности выше для мембраны УПМ-К и ниже для мембраны УПМ-100

Ключевые слова: идентификация, электронно-микроскопическое исследование, методика, поры, ультрафильтрационная мембрана, морфология, коэффициент засоренности.

\section{Введение}

На процесс массопереноса через ультрафильтрационные мембраны оказывает влияние несколько факторов: электрический потенциал, перепад давления на мембране, концентрация растворенного вещества и др. $[1,2]$. Кинетические ха- 
рактеристики (удельный выходной поток, коэффициент задержания, коэффициент диффузионной проницаемости) мембран при разделении растворов связаны не только с воздействием внешних параметров на мембранную систему, но и с количеством пор и их распределением по радиусам. При этом актуальным является разработка перспективных методик оценки распределения пор по радиусам и оценка микроструктуры поверхности для той или иной мембранной перегородки. В классическом варианте подобные методики связаны с использованием электронной микроскопии. В статье [3] показано изменение микроструктуры гетерогенных ионообменных мембран с повышением температуры с использованием метода растровой электронной микроскопии. Повышение пористости и доли проводящей фазы в объеме мембраны является следствием повышения влагоемкости мембраны при снижении обменной емкости. Авторами работы [4] проведены исследования поверхностной микроструктуры катионнообменной мембраны, выявившие изменение структуры в сторону уплотнения. На поверхности и в объеме мембраны отмечено снижение количества и размера микропор в результате взаимодействия $\mathrm{c}$ аминокислотой фенилаланином.

В работе [5] отмечается снижение транспортных свойств катионообменной мембраны МК-40 в результате осадкообразования и изменения морфологии поверхности при длительном использовании в режиме реверсного электродиализа. Микроскопические исследования структурных характеристик гетерогенных ионообменных мембран МК-40 и МА-40 после использования в электродиализаторе, проведенные авторами работы [6], выявили рост макропористости, а также снижение обменной емкости и селективности из-за образования осадков на поверхности и в объеме мембран. В литературе [7] методом растровой электронной микроскопии проведен сравни- тельный анализ структурных свойств поверхности экспериментальных катионообменных мембран Ralex CM Pes производства «MEGA» a.s. (Чехия). Выявлено влияние технологии изготовления на неоднородность поверхности и изменение размеров пор и доли дефектов структур. Неизменным остается соотношение проводящих и непроводящих фаз на поверхности мембран. В статье [8] отмечена возможность использования сканирующей электронной микроскопии для анализа структуры полимерных нанофильтрационных мембран.

На основании использования двух методов микроскопии, которые позволяют проводить трехмерную реконструкцию (сфокусированный ионный пучок и последовательная сканирующая электронная микроскопия) для анализа ультрафильтрационных пористых мембран авторами работы [9] предложен новый алгоритм оценки пористости и среднего размера пор в различных слоях. В работе [10] рассмотрены результаты применения нескольких методов микроскопии для получения поверхностных и объемных характеристик плоских мембран из полиэфирсульфона и полого волокна. В [11] с помощью сканирующей электронной микроскопии получены данные о влиянии различных композиций (поливиниловый спирт и крахмал) на пористые структуры полимерных мембран. В зависимости от содержания крахмала фиксируется образование микропор размером 1-10 мкм в полимерной мембране. В работе [12] предложено использование электронной микроскопии при исследовании морфологии ионных кластеров, образующихся в мембранах и влияющих на транспортные и электрические свойства. Авторами работы [13] проведены исследования конфигурации ячейки и ионной проводимости сульфированных мембран на основе полиэфиркетона с различной степенью сульфирования методом сканирующей электронной микроскопии. Проанализированы характеристики поверх- 
ности мембран и возможные механические повреждения во время измерения сопротивления.

В [14] отмечается, что для оценки изготовленных мембран при добавлении ПАВ адекватным является исследование поверхности мембран методом сканирующей электронной микроскопии. В paботе [15] уделяется внимание двухслойным наномембранам, в которые введены нанослои полимера для преодоления хрупкости. Методом сканирующей электронной микроскопии подтверждается отсутствие значительных дефектов при высокой гибкости наномембраны в результате действий макроскопических манипуляций.

Анализ работ [3-15] показывает, что в настоящее время микроскопические методы исследования поверхности, микроструктуры и распределения пор на поверхности мембран являются перспективными.

Современные программы для обработки изображений поверхности, такие как Image Expert Pro 3, Image Expert Sample 2, Image Expert Gauge, Image Expert 3D, как правило, предназначены для анализа металлических, графитовых включений, зеренной структуры, определения количества альфа-фазы, микроструктуры сталей [16]. Однако эти программы имеют существенный недостаток, так как они не представляют возможность работать с новыми образцами плоских цифровых изображений, и выступают только лишь как демонстраторы заложенных в эти программные продукты эталонов сравнения.

Встречаются также систематические работы по обработке цифровых изображений композитных полимерных мембран, например, [17-19]. Программные продукты этого типа обладают несомненными преимуществами: кооперация (возможность использования этих продуктов на этапе обмена информацией между родственными научными направления образовательных организаций и НИИ); адекватность обработки информации по поверхностной морфологии объектов ионообменных мембран. Но существует и специфическая особенность, заключающаяся в применении этого программного продукта, например, для анализа доли и размеров микрофаз, в частности пор.

Настоящая статья является продолжением работы, посвященной электронномикроскопическим исследованиям распределения пор по диаметрам на сорбционной поверхности ультрафильтрационных мембран. Первые результаты, полученные с применением подобного подхода, стандартных средств обработки данных Microsoft Excel 2010, AutoCad 2018 опубликованы в статье [20]. В настоящей статье предложен новый способ обработки тех же экспериментальных данных (СЭМ-изображений), сочетающий электронно-микроскопические исследования, обработку изображений Otsu's method и программную реализацию в Matlab 2017 [21], но получены новые результаты по идентификации пор на поверхности ультрафильтрационных мембран различных типов.

На основе сравнения достоинств и недостатков всего многообразия проанализированных методов, способов, приемов, прикладных программ, было принято решение о разработке программной реализации исследования свойств поверхности именно полимерных пористых мембран с использованием разработанного программного продукта, позволяющего существенно сократить время эксперимента [21]. Целью работы является разработка способа идентификации пор и их распределения на поверхности ультрафильтрационных мембран, заключающегося в обработке экспериментальных данных электронно-микроско-пических исследований Otsu's методом и программной реализации в Matlab 2017.

\section{Экспериментальная часть}

Объектами экспериментальных исследований являлись ультрафильтрационные мембраны УАМ-50, УАМ-100, 


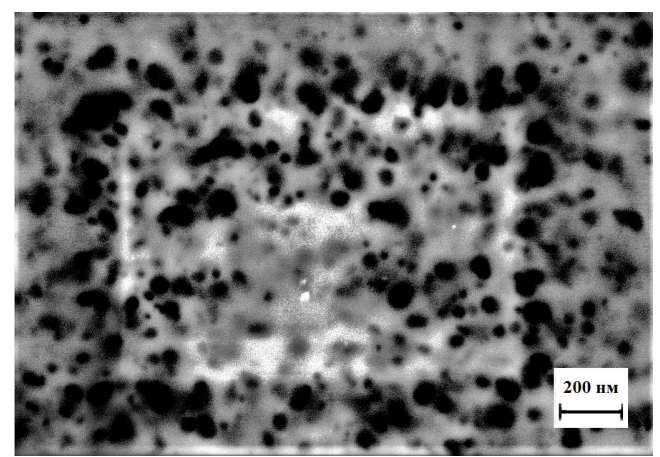

a

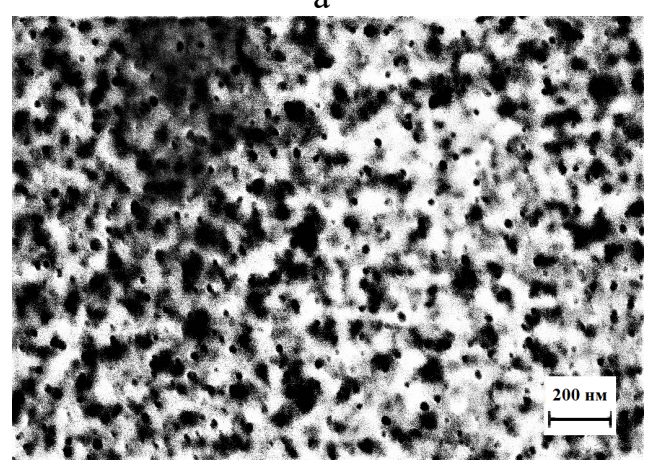

B

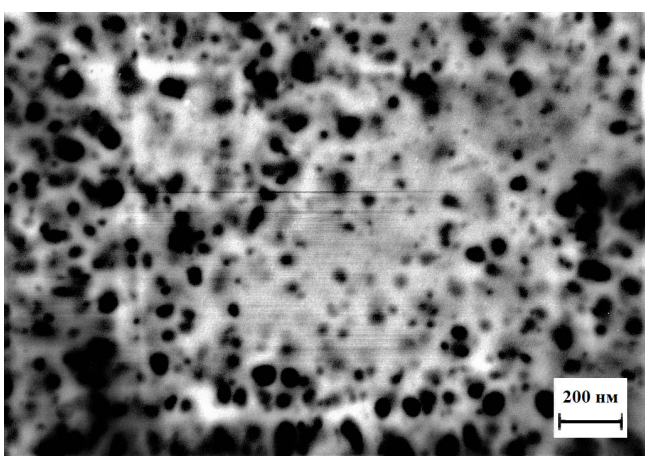

6

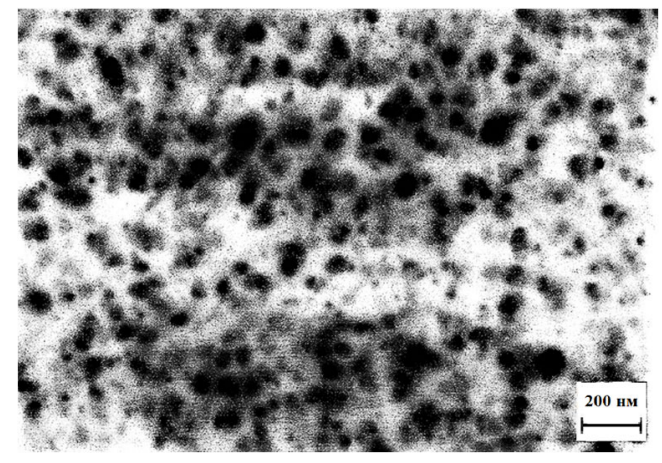

$\Gamma$

Рис. 1. Обработанные изображения выделенных участков поверхности ультрафильтрационных мембран УАМ-50 (а), УАМ-100 (б), УПМ-К (в), УПМ-100 (г) при увеличении 100 и ускоряющем напряжении $5 \mathrm{kV}$ с применением программного комплекса [21]

Fig. 1. Processed images of selected areas of the surface of ultrafiltration membranes (a) UAM-50, (b) UAM-100, (c) UPM-K, (d) UPM-100 at a magnification of 100 and an accelerating voltage of $5 \mathrm{kV}$ using the software package [21]

УПМ-К, УПМ-100, характеристики и показатели которых подробно представлены в литературе $[22,23]$, а исследование распределения пор по диаметрам на поверхности мембран в работе [20].

Обработку изображений выделенных участков поверхности исследуемых ультрафильтрационных мембран и расчет коэффициента засоренности производили при помощи программного комплекса [21] в пакете Matlab 2017.

Методика исследования и расчета состояла в следующем.

Заранее выбранные для эксперимента сухие образцы мембран УАМ-50, УАМ100 УПМ-К, УПМ-100 подвергались исследованию (визуализация поверхности с помощью электронного микроскопа Merlin «CarlZeiss», Германия). Далее проводилась процедура фиксации изображения поверхности мембраны с помощью программы анализа изображений Axiovision (рис. 1).
Выделенные участки ультрафильтрационных мембран площадью $1000000 \mathrm{м}^{2}$ обрабатывались при помощи Matlab 2017 для получения основных параметров, таких как средний диаметр засоренности (диаметры пор и коэффициент засоренности мембран). При обработке больших массивов данных по средним диаметрам пор и коэффициенту засоренности мембран использовался ПК, который позволил снизить и рассчитать погрешность выполненных измерений при помощи стандартных методов математической статистики.

Обработка изображения мембран УАМ-50, УАМ-100, УПМ-К, УПМ-100 проводилась автоматизированным методом (рис. 2).

Сравнивали черно-белые изображения с бинарными (двоичными) (рис. 3) и оценивали их достоверность. Дополнительно проводилось визуальное сравнение этих изображений (черно-белого и бинарного). Эта процедура необходима из-за 


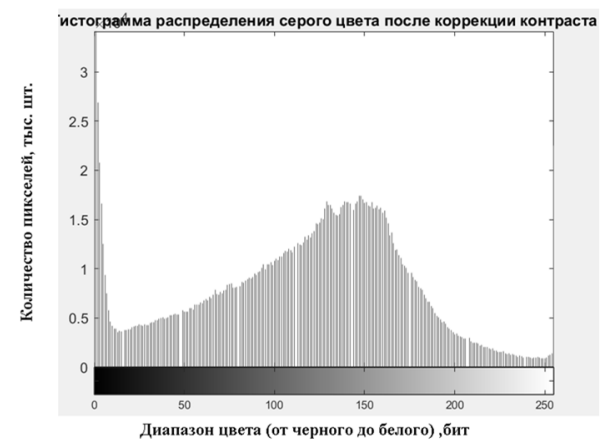

a

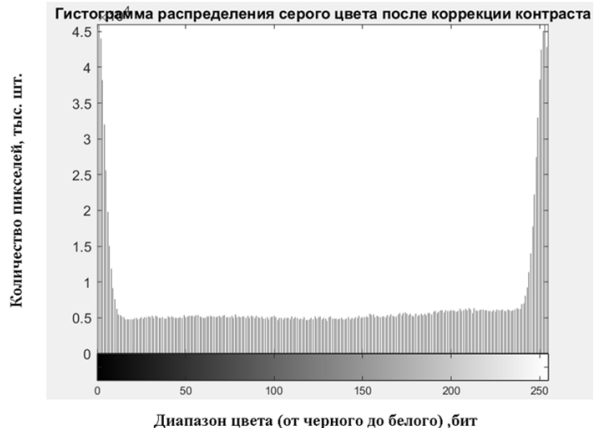

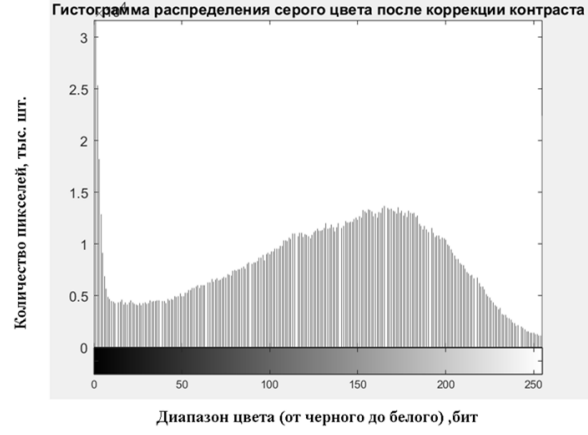

б

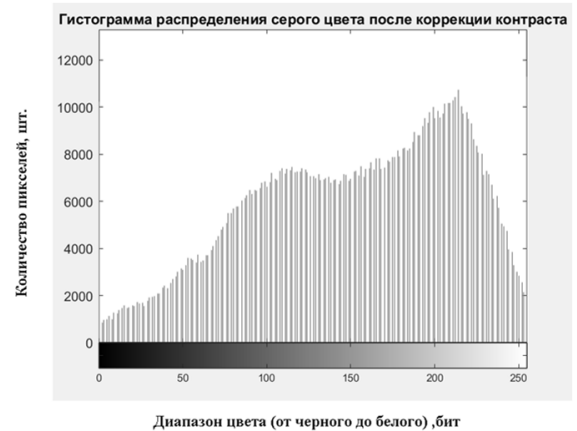

Рис. 2. Гистограмма распределения цвета после коррекции мембран УАМ-50 (a), УАМ-100 (б), УПМ-К (в), УПМ-100 (г)

Fig. 2. Histogram of colour distribution after correction of UAM-50 (a), UAM-100 (b), UPM-K (c), UPM-100 (d) membranes

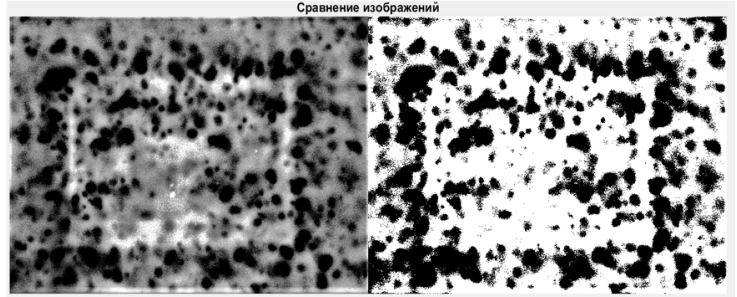

a

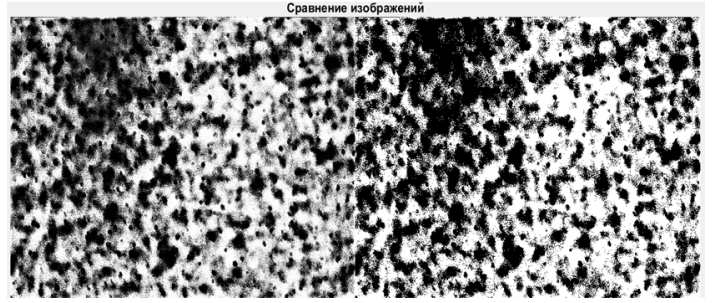

B

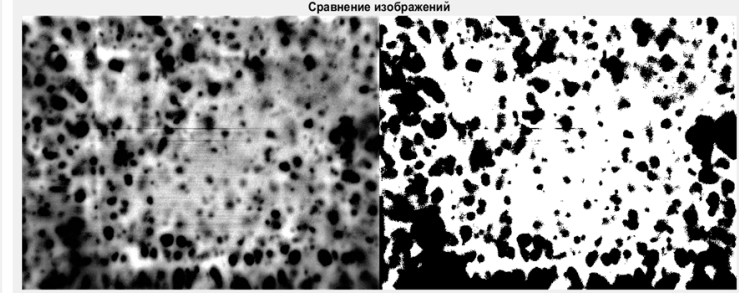

6

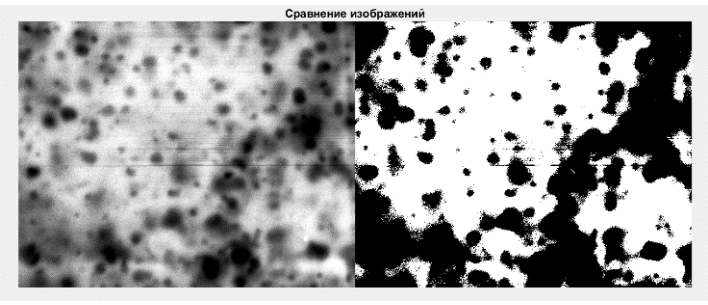

$\Gamma$

Рис. 3. Сравнение чёрно-белого изображения с бинарным мембран УАМ-50 (а), УАМ-100 (б), УПМ-К (в), УПМ-100 (г)

Fig. 3. Comparison of a black-and-white image with binary membranes UAM-50 (a), UAM-100 (b), UPM-K (c), UPM-100 (d)

того, что в практических случаях значение порога метода Otsu's (в автоматическом режиме) не всегда получается достоверным из-за свойств яркости изображения или шумов. Поэтому в случае недостаточно контрастного изображения значение порога устанавливалось вручную.

Для разделения изображения на цвета использовались фильтры - красный, зелёный и синий. Разделение их по отдельно- 
сти при наложении друг на друга позволяет получать более точное бинарное изображение.

Работа программы Matlab 2017 сопряжена с работой в белом цвете, для чего
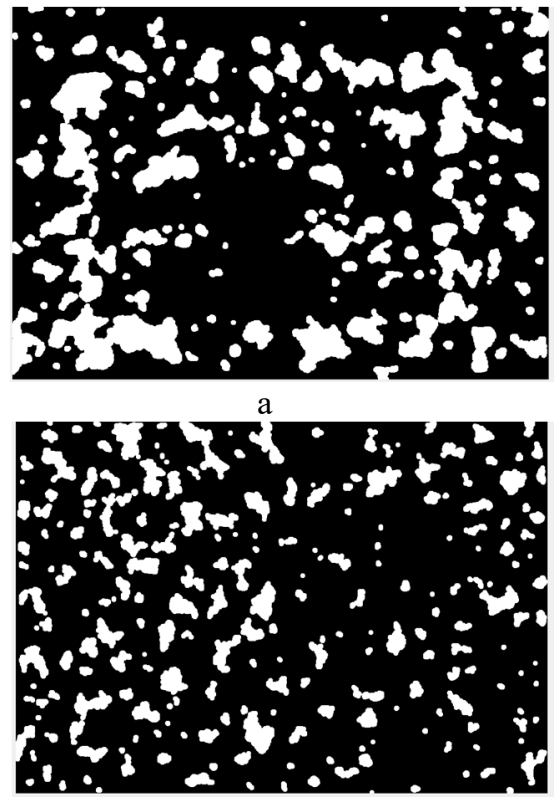

B

Рис. 4. Морфологически обработанное изображение для мембран УАМ-50 (а), УАМ-100 (б), УПМ-К (в), УПМ-100 (г)

Fig. 4. Morphologically processed image for UAM-50 (a), UAM-100 (b), UPM-K (c), UPM-100 (d) membranes
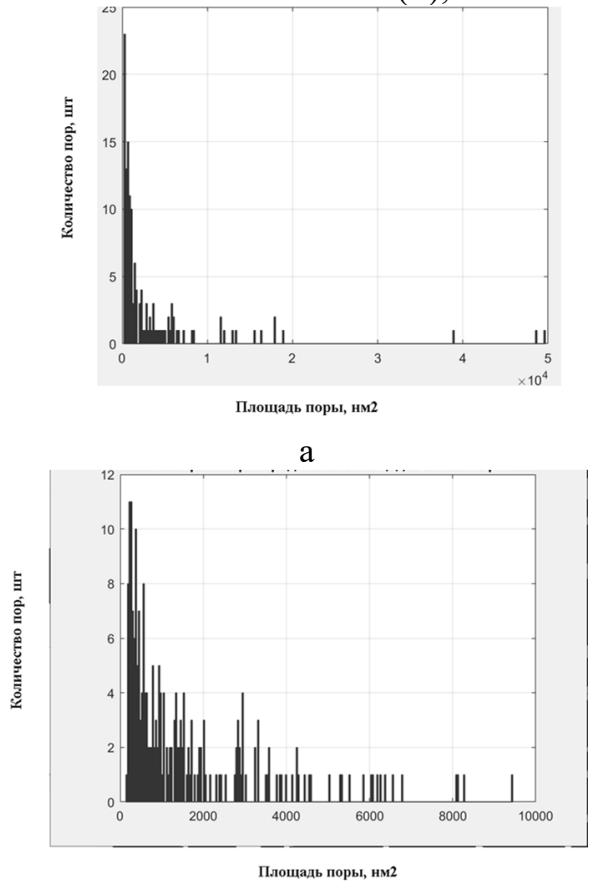

B

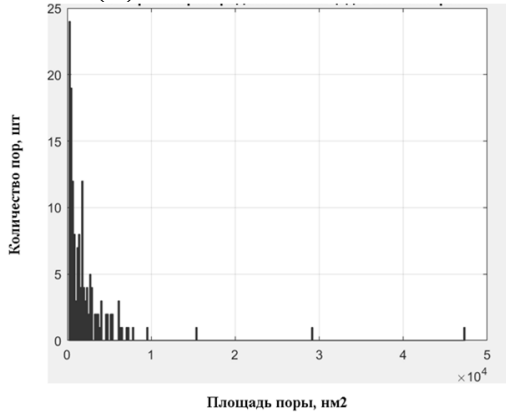

б

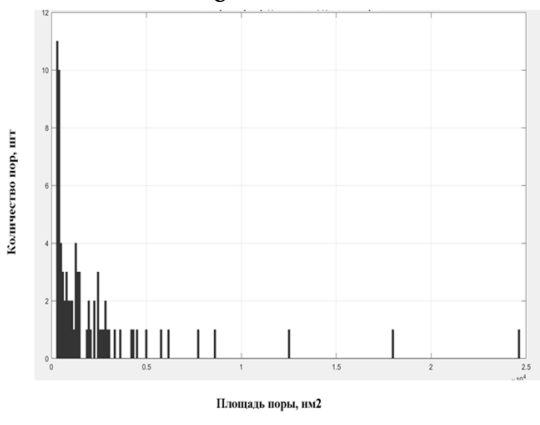

$\Gamma$ полученное суммарное изображение переводили в чёрный фон с помощью программы Matlab 2017 для получения окружностей пор мембран (рис. 4).

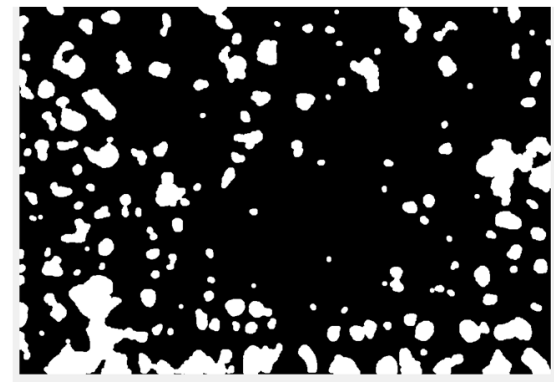

6

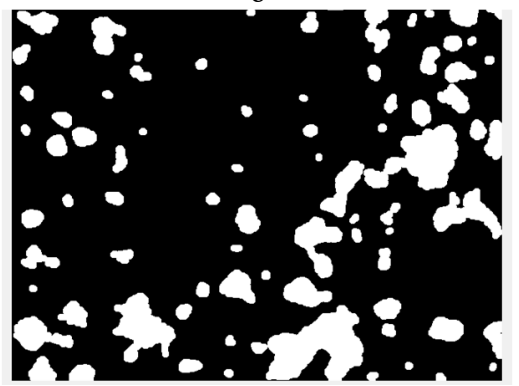

Рис. 5. Гистограмма распределения количества объектов от площадей для мембран УАМ-50 (а), УАМ-100 (б), УПМ-К (в), УПМ-100 (г)

Fig. 5. Histogram of the distribution of the number of objects versus areas for UAM-50 (a), UAM-100 (b), UPM-K (c), UPM-100 (d) membranes 
При помощи стандартных функций Matlab 2017 определяются площади объектов на бинарном изображении, причем сохранение их производится в виде массива со значениями площади. Далее строится гистограмма распределения площадей с определённым шагом в зависимости от количества полученных данных (рис. 5)

\section{Обсуждение результатов}

Визуальный анализ выделенных участков поверхности ультрафильтрационных мембран УАМ-50, УАМ-100, УПМ-К, УПМ-100 (рис. 1) показал не очень контрастный характер изображения (особенно для мембраны УПМ-100). Для устранения этого недостатка и для создания контрастного изображения строились гистограммы распределения цвета: от 0 - (черный фон) до 256 (белый фон) (рис. 2).

Проведенный анализ полученных гистограмм распределения показал, что наибольшее сосредоточение цветового распределения установлено для мембран УАМ-50, УАМ-100 в диапазоне 144...170 бит и УПМ-К, УПМ-100 в диапазоне 220. . .250 бит.

В пакете Matlab 2017 используется функция получения более контрастного изображения выделенных участков. Поэтому получаемое изображение имеет более резкие переходы между засоренной и чистой частью. Гистограммы (рис. 2) показывают исходный диапазон яркостей от минимально возможной до максимальной.
Для оценки погрешности и надежности определения количественных характеристик морфологии поверхности анализировали пять электронных изображений, полученных для различных участков поверхности исследуемой мембраны. Процедуру обработки каждого электронного изображения образца повторяли десять раз на основании рекомендаций, представленных в работе [24]. Статистическая обработка результатов показала, что относительное стандартное отклонение не превышало 0.1 .

Коэффициент засоренности мембраны определяется с помощью формулы (1):

$$
k=\frac{\sum S_{\text {неон }}}{\sum S_{n}},
$$

где $S_{\text {неодн }}-$ площадь пор на изображении, мкм $^{2} ; S_{n}$ - площадь исследуемого изображения, мкм².

C помощью функции нахождения среднего значения определяли среднюю площадь пор поверхности мембраны и средний диаметр пор по формуле (2):

$$
D_{\text {cp }}=\sqrt{\frac{4 \cdot S_{\mathrm{cp}}}{\pi}},
$$

где $D_{\text {ср }}-$ средний диаметр пор, нм; $S_{\mathrm{cp}}$ - полученная в результате обработки изображений средняя площадь пор мембран, нм$^{2}$.

Расчетные данные по количеству объектов, средней площади пор поверхности мембраны, среднего диаметра пор мембран, полученные с использованием разработанной методики приведены в таблице 1.

Таблица 1. Расчетные данные для ультрафильтрационных мембран УАМ-50, УАМ-100, УПМ-К, УПМ-100

Table 1. Calculated data for ultrafiltration membranes UAM-50, UAM-100, UPM-K, UPM-100

\begin{tabular}{|l|c|c|c|c|}
\hline \multicolumn{1}{|c|}{ Тип мембраны } & УАМ-50 & УАМ-100 & УПМ-К & УПМ-100 \\
\hline Количество объектов & 140 & 149 & 213 & 102 \\
\hline $\begin{array}{l}\text { Средняя площадь объ- } \\
\text { екта, нм }{ }^{2} \cdot 10^{3}\end{array}$ & 2.87 & 2.47 & 1.97 & 2.06 \\
\hline Средний диаметр, нм & $60 \pm 3$ & $56 \pm 3$ & $51 \pm 2$ & $51 \pm 2$ \\
\hline $\begin{array}{l}\text { Коэффициент засорен- } \\
\text { ности }\end{array}$ & 0.19 & 0.17 & 0.20 & 0.16 \\
\hline
\end{tabular}


Анализ данных таблицы 1 показывает, что средняя площадь объекта минимальна для мембраны УПМ-К и максимальна для мембраны УАМ-50. Средний диаметр пор поверхности исследуемых мембран находится в интервале от 51 до 60 нм. При этом коэффициент засоренности выше для мембраны УПМ-К и ниже для мембраны УПМ-100. Полученные по разработанной методике расчетные данные среднего диаметра пор мембран (не прошедших обработку в экспериментальной ячейке под давлением при ультрафильтрации) сопоставимы с данными работы [20] для ультрафильтрационных мембран УАМ-50, УАМ-100, УПМ-К, УПМ-100, на поверхности которых средний диаметр пор находится в интервале от 54 до 70 нм.

\section{Заключение}

Разработанный метод позволяет автоматизировано рассчитывать количество, среднюю площадь, диаметр пор на поверхности и коэффициент засоренности полимерных ультрафильтрационных мембран. На основе предлагаемого метода, сочетающего электронно-микроскопические исследования, обработку изображений Otsu's method и программную реализацию в Matlab 2017 [21] доказана возможность получения достоверных и воспроизводимых данных по анализу морфологии поверхности ультрафильтрационных мембран на основе статистической обработки большой выборки экспериментальных данных.

\section{Микрофотографии получены в Центре коллективного пользования научным оборудованием ТГУ им. Г.Р. Державина.}

\section{Список литературы}

1. Васильева В.И., Голева Е.А., Селеменев В.Ф., Карпов С.И. и др. // Журнал физической химии. 2019. Т. 93. № 3. С. 428-437.

2. Лазарев С.И., Ковалева О.А., Коновалов Д.Н., Игнатов Н.Н. // Поверхность. Рентгеновские, синхротронные и нейтронные исследования. 2021. № 3. С. 1-8.

3. Акберова Э.М., Колганов В.И., Коротков Д.В., Бабичев С.В. // Сорбиионные и хроматографические прочессы. 2016. Т. 16. № 5. C. 631-639.

4. Голева Е.А., Васильева В.И., Селеменев В.Ф., Кузнецов В.А. и др. // Сорбиионные $и$ хроматографические прочессы. 2016. Т. 16. № 5. C. 640-652.

5. Яцев А.М., Акберова Э.М., Голева Е.А., Васильева В.И. и др. // Сорбиионные и хроматографические прочессы. 2017. Т. 17. № 2. C. 313-322.

6. Акберова Э.М., Васильева В.И., Смагин М.А., Костылев Д.В. // Сорбиионные и хроматографические прочессы. 2019. Т. 19. № 4. C. $434-442$.

7. Акберова Э.М., Васильева В.И., Костылев Д.В., Смагин М.А. // Сорбиионные и хроматографические прочессы. 2019. Т. 19. № 5. C. 557-565.
8. Kowalik-Klimczak A., Bednarska A., Gradkowski M. // Problemy eksploatacji - maintenance problems. 2016. Vol. 1. pp. 119-128.

9. Sundaramoorthi G., Hadwiger M., Ben Romdhane M., Behzad A.R. et al. // Industrial \& Engineering Chemistry Research. 2016. Vol. 55. I. 12. pp. 3689-3695.

10. Tamime R., Wyart Y., Siozade L., Baudin I. et al. // Membranes (Basel). 2011. Vol. 1 (2). pp. 91-97.

11. Zhang Y., Zhou L., Mao E. // Applied Mechanics and Materials. 2011. Vol. 109. pp. 110113.

12. Yakovlev S., Downing K.H. // Phys. Chem. Chem. Phys. 2013. Vol. 15. pp. 1052-1064.

13. Fedorenko D., Vaivars G. // Functional Materials and Nanotechnologies. Conf. Series: Materials Science and Engineering. 2019. Vol. 503. pp. 1-5.

14. Arahman N., Maimun T., Syawaliah M. // AIP Conference Proceedings. 2017. Vol. 1788. pp. 1-7.

15. Mersha A., Selyanchyn R., Fujikawa Sh. // Clean Energy. 2017. Vol. 1. No 1. pp. 80-89.

16. Модификатор: сайт ИЦ Модификатор: [Электронный ресурс]. Дата обновления: 04.05.2018. URL: http://www.modificator.ru/ $\mathrm{ad} /$ nexsys.html (дата обращения: 04.05.2018). 
17. Сирота Е.А., Кранина Н.А., Васильева В.И. // Свидетельство о государственной регистрации программы для ЭВМ № 2012610185.

18. Akberova E.M., Vasil'eva V.I. // Electrochemistry Communications. 2020. Vol. 111. No 106659. pp. 1-6.

19. Akberova E.M., Vasil'eva V.I., Zabolotsky V.I., Novak L. // Membranes. 2019. Vol. 9. No 169. pp. 1-13.

20. Лазарев С.И., Ковалев С.В., Коновалов Д.Н., Рыжкин В.Ю. и др. // Сорбционные и хроматографические прочессы. 2020. Т. 20. № 6. C. 707-718.
21. Лазарев С.И., Рыжкин В.Ю., Ковалева О.А., Головин Ю.М. и др. // Свидетельство о государственной регистрации программы для ЭВМ № 2018611402.

22. Мембраны, фильтрующие элементы, мембранные технологии. Каталог. Владимир: ЗАО НТЦ «Владипор». 2004. 22 с.

23. Бонн А.И., Дзюбенко В.Г., Шишова И.И. // Высокомолекулярные соединения. Серия Б. 1993. Т. 35. № 7. С. 922-932.

24. Васильева В.И., Акберова Э.М., Жильцова А.В., Черных Е.И. и др. // Поверхность. Рентгеновские, синхротронные и нейтронные исследования. 2013. № 9. С. 27-34.

\title{
Identification of pores of ultrafiltration membranes based on electron microscopic studies
}

\author{
(C) 2021 Lazarev S.I. ${ }^{1}$, Konovalov D.N. ${ }^{1}$, Kovalev S.V. ${ }^{1,2}$, Ryzhkin V.Yu. ${ }^{1}$, \\ Polyansky K.K. ${ }^{3}$, Kovaleva O.A. ${ }^{1,2}$ \\ ${ }^{I}$ Tambov State Technical University, Tambov \\ ${ }^{2}$ Derzhavin Tambov State University, Tambov \\ ${ }^{3}$ Voronezh Branch of Plekhanov Russian University of Economics, Voronezh
}

The study analyses methods, modes, techniques, and applications for the identification of pores in polymer membranes. Based on a comparison of the advantages and disadvantages of the methods, an approach to the development of the software for the study of the pores of polymeric semipermeable membranes is proposed. The objects of the study were UAM-50, UAM-100, UPM-K, and UPM-100 type ultrafiltration membranes, the choice of which was provided by high retention capacity, good performance and the high applicability in industrial practice. A method for the calculation of the membrane contamination coefficient, allowing to determine the period of effective operation of ultrafiltration membranes, elements and installations for baromembrane and electrobaromembrane separation, concentration and purification of industrial solutions and effluents is presented. Selected sections of ultrafiltration UAM-50, UAM-100, UPM-K, and UPM-100 membranes with an area of $1.000 .000 \mathrm{~nm}^{2}$ were processed using Matlab 2017 in such a way that the main parameters, such as the average contamination diameter (pore diameters and membrane contamination factor) were obtained. For processing large data sets on the average pore diameters and the membrane contamination coefficient, a PC was used. The use of PC allowed to reduce and calculate the error of the measurements performed using standard methods of mathematical statistics. The calculation of the membrane contamination coefficient was carried out using a program studying the description of the main functions of the imaging processing toolbox. The developed method significantly reduced the time of the experiment and allowed automatically calculate the number of objects, average area, and pore diameter on the sorption surface. The method combining electron microscopic studies, image processing using Otsu's method, software implementation in Matlab 2017, provides possibility to obtain reliable and reproducible data on the surface morphology of ultrafiltration UAM-50, UAM-100, UPM-K, and UPM-100 membranes, based on statistical processing of a large sample of data obtained as a result of electron microscopic studies.

Analysis of the experimental data obtained by the automated method showed that the average area of the object was the smallest for the UPM-K membrane and the largest for the UAM-50 membrane, and the average pore diameter of the surface of the studied membranes was in the range from 51 to $60 \mathrm{~nm}$, which was comparable with results obtained by other methods and described in the literature. At the same time, the contamination coefficient was higher for the UPM-K membrane and lower for the UPM-100 membrane.

Keywords: ultrafiltration membrane, electron microscopic examination, pores, morphology, clogging factor. 


\section{References}

1. Vasil'eva V.I., Goleva E.A., Selemenev V.F., Karpov S.I. et al., Russian Journal of Physical Chemistry A, 2019, Vol. 93, No 3, pp. 542-550.

2. Lazarev S.I., Kovaleva O.A., Konovalov D.N., Ignatov N.N., Poverkhnost. Rentgenovskiye, sinkhrotronnye i neytronnye issledovaniya, 2021, No 3, pp. 1-8.

3. Akberova E.M., Kolganov V.I., Korotkov D.V., Babichev S.V., Sorbtsionnye i khromatograficheskiye protsessy, 2016, Vol. 16, No 5, pp. 631-639.

4. Goleva E.A., Vasil'eva V.I., Selemenev V.F., Kuzneczov V.A. et al., Sorbtsionnye $i$ khromatograficheskiye protsessy, 2016, Vol. 16, No 5, pp. 640-652.

5. Yacev A.M., Akberova E.M., Goleva E.A., Vasil'eva V.I. et al., Sorbtsionnye i khromatograficheskiye protsessy, 2017, Vol. 17, No 2, pp. 313-322.

6. Akberova E.M., Vasil 'eva V.I., Smagin M.A., Kostylev D.V., Sorbtsionnye i khromatograficheskiye protsessy, 2019, Vol. 19, No 4, pp. 434-442.

7. Akberova E.M., Vasil'eva V.I., Kostylev D.V., Smagin M.A., Sorbtsionnye i khromatograficheskiye protsessy, 2019, Vol. 19, No 5, pp. 557-565.

8. Kowalik-Klimczak A., Bednarska A., Gradkowski M., Problemy eksploatacji - maintenance problems, 2016, Vol. 1, pp. 119-128.

9. Sundaramoorthi G., Hadwiger M., Ben Romdhane M., Behzad A.R. et al., Industrial \& Engineering Chemistry Research, 2016, Vol. 55, I. 12, pp. 3689-3695.

10. Tamime R., Wyart Y., Siozade L., Baudin I. et al., Membranes (Basel), 2011, Vol. 1 (2), pp. 91-97.

11. Zhang Y., Zhou L., Mao E., Applied Mechanics and Materials, 2011, Vol. 109, pp.110-113.

Лазарев Сергей Иванович - профессор кафедры «Механика и инженерная графика», д.т.н., Тамбовский государственный технический университет, Тамбов

Коновалов Дмитрий Николаевич - доцент кафедры «Техника и технологии автомобильного транспорта», к.т.н., Тамбовский государственный технический университет, Тамбов

Ковалев Сергей Владимирович - профессор кафедры «Механика и инженерная графика», д.т.н., Тамбовский государственный технический университет, Тамбов; профессор кафедры
12. Yakovlev S., Downing K.H., Phys. Chem Chem. Phys, 2013, Vol. 15, pp. 1052-1064.

13. Fedorenko D., Vaivars G., Functional Materials and Nanotechnologies. Conf. Series: Materials Science and Engineering, 2019, Vol. 503, pp. 1-5.

14. Arahman N., Maimun T., Syawaliah M., AIP Conference Proceedings, 2017, Vol. 1788, pp. 1-7.

15. Mersha A., Selyanchyn R., Fujikawa Sh., Clean Energy, 2017, Vol. 1, No 1, pp. 80-89.

16. Modifikator: sajt ICz Modifikator: [E'lektronny`j resurs], Data obnovleniya: 04.05.2018, URL: http://www.modificator.ru/ad/nexsys. html (data obrashheniya: 04.05.2018).

17. Sirota E.A., Kranina N.A., Vasil’eva V.I., Svidetelstvo o gosudarstvennoj registracii programmy dlya EVM № 2012610185.

18. Akberova E.M., Vasil'eva V.I., Electrochemistry Communications, 2020, Vol. 111, No 106659, pp. 1-6.

19. Akberova E.M., Vasil'eva V.I., Zabolotsky V.I., Novak L., Membranes, 2019, Vol. 9, No 169, pp. 1-13.

20. Lazarev S.I., Kovalev S.V., Konovalov D.N., Ryzhkin V.YU. et al., Sorbtsionnye i khromatograficheskiye protsessy, 2020, Vol. 20, No 6, pp. 707-718.

21. Lazarev S.I., Ryzhkin V.Yu., Kovaleva O.A., Golovin Yu.M. et al., Svidetelstvo o gosudarstvennoj registracii programmy dlya EVM No 2018611402.

22. Membrany, filtruyushhie elementy, membrannye texnologii, Katalog, Vladimir, ZAO NTCz «Vladipor», 2004, 22 p.

23. Bonn A.I., Dzyubenko V.G., Shishova I.I., Vysokomolekulyarnye soedineniya. Seriya $B$, 1993, Vol. 35, No 7, pp. 922-932.

24. Vasil`eva V.I., Akberova E’.M., Zhilczova A.V., Chernyx E.I. et al., Poverkhnost. Rentgenovskiye, sinkhrotronnye i neytronnye issledovaniya, 2013, No 9, pp. 27-34.

Lazarev Sergey I. - Professor Department of Mechanics and Engineering Graphics, Doctor of Technical Sciences, Tambov State Technical University, Tambov

Konovalov Dmitriy N. - Associate Professor of the Department of Engineering and Technology of Road Transport, Ph.D., Tambov State Technical University, Tambov

Kovalev Sergey V. - Professor Department of Mechanics and Engineering Graphics, Doctor of Technical Sciences, Tambov State Technical University, 
математического моделирования и информационных технологий, д.т.н., Тамбовский государственный университет имени Г.Р. Державина, Тамбов

Рыжкин Владимир Юрьевич - аспирант, Тамбовский государственный технический университет, Тамбов

Полянский Константин Константинович профессор кафедры «Коммерции и товароведения», д.т.н., Воронежский филиал Российского экономического университета имени Г.В. Плеханова, Воронеж

Ковалева Ольга Александровна - профессор кафедры математического моделирования и информационных технологий, д.т.н., Тамбовский государственный университет имени Г.Р. Державина, Тамбов; доцент кафедры «Экономика», д.т.н., Тамбовский государственный технический университет, Тамбов
Tambov; Professor of the Department of Mathematical Modeling and Information Technologies, Doctor of Technical Sciences, Derzhavin Tambov State University, Tambov

Ryzhkin Vladimir Yu. - Student, Tambov State Technical University, Tambov

Polyansky Konstantin K. - Professor of the Department of Commerce and Commodity Science, Doctor of Technical Sciences, Voronezh Branch of the Russian Economic University named after G.V. Plekhanov, Voronezh

Kovaleva Olga A. - Professor of the Department of Mathematical Modeling and Information Technologies, Doctor of Technical Sciences, Derzhavin Tambov State University, Tambov; Associate Professor Department of Economy, Doctor of Technical Sciences, Tambov State Technical University, Tambov 